\title{
Bilateral elbow indirect Cubital tunnel syndrome due to mass effect of Gouty tophi
}

\begin{abstract}
Gout is an inflammatory arthritis associated with raised uric acid levels in blood. The pathology involves infiltration and destruction of ligament, cartilage, tendons, bone and skin by deposition of urate crystals. Our purpose of writing this case is to report rare bilateral neurological symptoms due to compression, by tophaceous gout around elbows. A 32-year-old male had presented with episodic tingling in bilateral ulnar nerve distributions for fourteen days. Surgical excision of the tophi around elbows that were causing the neural compression symptoms was done and symptoms relieved gradually over 6 weeks. Tophi are late presentations. They can present with symptoms from depositing at different sites that is evidenced in existing literature case reports. Controlling serum uric acid levels below deposition threshold either by dietary or pharmaceutical drugs is the mainstay of management, resulting in dissolution of monosodium urate crystals.
\end{abstract}

Volume 13 Issue 4 - 202 I

\author{
Akshay Lekhi,Ashok Kumar \\ 'Depatment of Trauma and Orthopaedics, NHS, UK \\ ${ }^{2}$ Professor and Head of the department Orthopaedics, Saraswati \\ Medical College, India
}

Correspondence: Akshay Lekhi MBBS, MS Orth, DipSICOT, IOA fellow (Arthroscopy), Email akshaylekhi@gmail.com

Received: May 08, 202I | Published: July 21, 202

\section{Background}

The purpose of writing this case is to report rare bilateral neural symptoms due to tophaceous gout around elbows. Bilateral cubital tunnel syndrome due to tophaceous gout, has not been reported earlier. Gout is an inflammatory arthritis associated with raised uric acid levels in blood. The pathology involves infiltration and destruction of ligament, cartilage, tendons, bone and skin by deposition of urate crystals, with predilection for extensor surfaces accompanied by an acute or chronic inflammatory response. Controlling serum uric acid levels below deposition threshold either by dietary or pharmaceutical drugs is the mainstay of management, resulting in dissolution of monosodium urate crystals. ${ }^{1,2}$ A differential diagnosis of pseudogout, crystal deposition disorder and pseudotumor should be kept in consideration. There have been individual elbow swellings with tophi collection as the underlying cause reported in past ${ }^{3}$ but mass effect and neurological compression signs occurring bilaterally is a rare presentation in view of the existing literature evidence further detailed.

\section{Case presentation}

The 32-year-old male presented to outpatient clinic in 2016, with worsening paraesthesia in bilateral forearms and hands with gradually increasing swellings around the elbows (Figure 1). He was already diagnosed with chronic gout. Unfortunately, he could not continue the medications on a regular basis as prescribed by rheumatologists. The patient was a manual labourer by profession, otherwise fit and healthy, not allergic to any medications in past and non-respondent to the medical treatment since a fortnight; had presented with episodic tingling in ulnar nerve distributions for fourteen days. The paraesthesia in both the forearms was typical of a cubital tunnel syndrome and reproducible with flexion beyond 90 degrees. The paraesthesia was typically in the ring and little fingers of hand and occasional pain in the forearm. However the grip was not yet affected. The early signs of cubital tunnel syndrome were clinically an indication for decompression. There was occasional elbow pain associated with resting the elbow against hard surface to the grade $5 / 10$ on Visual analogue scale. There was no difficulty in joint movement from 0 to
90 degrees and bilateral grip was of normal strength. The symptoms had a gradual onset. There was a pain-free joint movement and no distal sensory or vascular deficits on examination with elbow at rest. Tophi were also observed on bilateral foot metatarsophalangeal joint area over the dorsum (both $2.5 \times 1.5 \mathrm{~cm}$, measure with measuring tape) and on the left Achilles tendon, measuring $1 \times 1.5 \mathrm{~cm}$, but remained symptomless (Figure 1). The fact that old serum uric acid level reports were pointing towards uncontrolled uric acid levels, recent clinical examination and a fine needle aspirate showing crystals, helped us to narrow down the differential diagnosis to uncontrolled tophaceous gout. The patient was managed by medications as per rheumatological advice and surgical excision of the tophi around elbows (both $\sim 3 \mathrm{x}$ $4 \mathrm{x} 2.5 \mathrm{~cm}$, measured with Vernier calliper and sterile measuring ruler) that were causing the neural compression symptoms. The tophi were external to the cubital tunnel but found compressing the nerve underneath and indirectly causing cubital tunnel compression neuropathy with mass effect. Hence both the cubital tunnels were also decompressed to release the pressure off the compressed nerve

After regular surgical follow up, dietary control and aggressive medical input, the symptomless tophi on foot also reduced in size substantially and there has been no recurrence in the last two years. The first follow up visit VAS for the occasional bilateral elbow pain was $1 / 10$, as the pressure effect had subsided and there was no pain or paraesthesia reproduced on resting the elbows on hard surface. The size of other tophi reduced by half over one year and since they were completely symptomless, no further surgical intervention was planned. The patient did have a change of medical dosage and occasional prescriptions of non-steroidal anti- inflammatory drugs for episodic pain post minor trauma at work, for up to 1 years post-surgery and was symptomless in the second follow up year. The patient did not turn up for follow up after two years.

The fact that chronic tophaceous growths around multiple joints may remain persistent for long time, despite pharmacological treatment, as in this case, demands a regular follow up for observation in such cases to prevent recurrence of tophi leading to compression signs. 

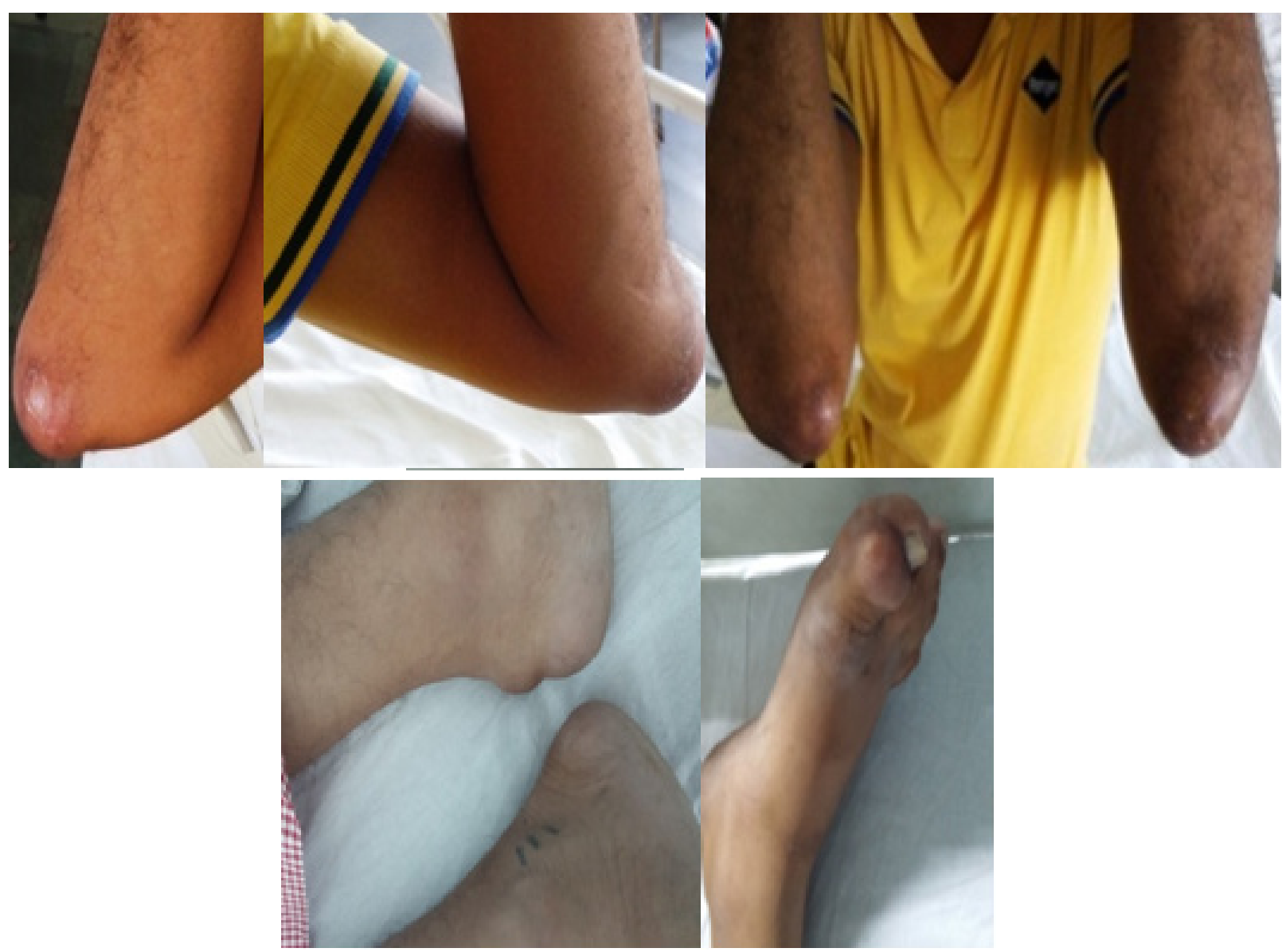

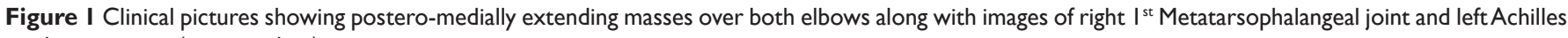
tendon insertion (symptomless).

\section{Investigations}

Clinical examination plays key role in streamlining and narrowing the differential diagnosis after the clinical history. Although there was a clear history of ignored gout treatment at patient's end, we could still not rule out the possibility of multiple soft tissue swellings around the elbow due to other differential diagnosis in mind as a systematic approach. Bilateral elbow radiographs were done as routine and a postero-medially extending shadow may be seen in the anteroposterior radiograph of right elbow (Figure 2). Fine needle aspiration cytology revealed chalky material- crystal deposits, surrounded by lymphocytes, giant cells and occasional red cells. Radiographs did not show any intra-articular involvement. Bilateral elbow joint aspirate revealed gouty crystals (monosodium urate, MSU) that is a pathognomonic feature. C- reactive protein was raised (80) and ESR (erythrocyte sedimentation rate) was 18 . The CRP levels gradually decline over 3 weeks to $<5$. There other significant finding in the full blood count.
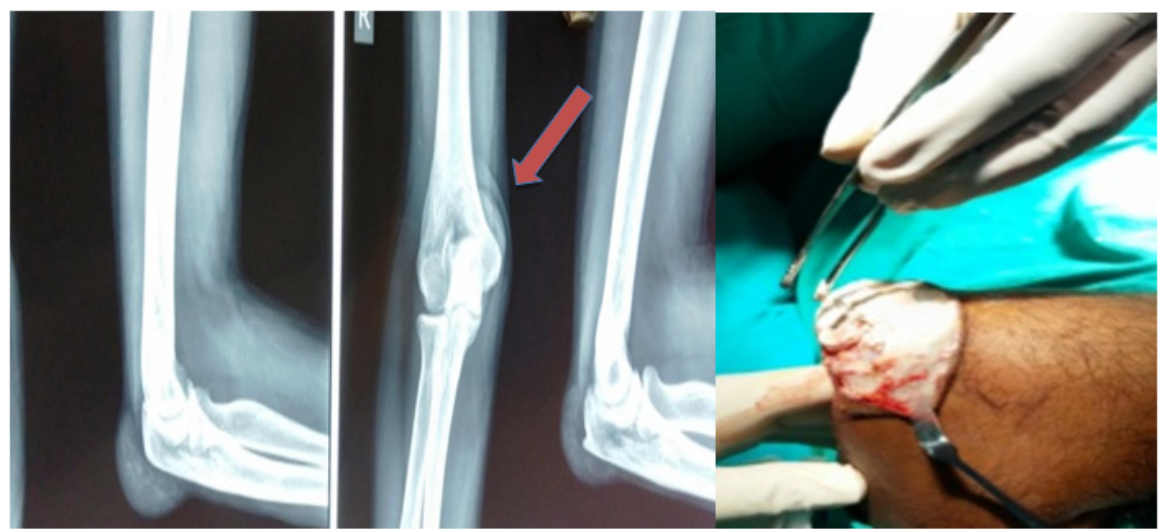

Figure 2 The arrow shows the mass extending postero-medially and compressing on the ulnar nerve causing patients paraesthesia symptoms. Posterior approach to dissect and expose the whole mass.

Citation: Lekhi A, Kumar A. Bilateral elbow indirect Cubital tunnel syndrome due to mass effect of Gouty tophi. MOJ Orthop Rheumatol. 202I;I3(4):70-73. DOI: 10.15406/mojor.202I.13.0055 I 


\section{Differential diagnosis}

The differential diagnosis kept in mind while approaching to the final diagnosis were chronic gout tophi, sarcomatous swelling, pseudogout swellings, aseptic bursitis or any soft tissue collection/ swelling acting as a pseudotumor. The diagnosis was based on clinical history of previously diagnosed hyperuricaemia, ignored regular medication intake, fine needle aspiration cytology and joint aspirations showing crystals and blood picture with high urate levels. Sarcomatous swelling is less likely to be bilateral or just around the joint and biopsy is the definitive investigation. Pseudogout preferentially occurs in large joints and less likely symmetrical. Joint aspiration reveals typical pseudogout crystals of calcium pyrophosphate which was not found in this case. Although the patient does not have fever and blood picture had normal white cell count, bursitis is less likely the diagnosis in this case as the swellings are hard to touch, bilateral and non-fluctuant. Hence we could narrow down the diagnosis with all these findings and finally reached to chronic gouty tophi as the diagnosis with fine needle aspiration and joint aspiration as preceding investigations and histopathological examination as the final confirmation.

\section{Treatment}

Both pharmacological and surgical interventions are important in this case scenario. Under appropriate anti-uricaemia medication (Allopurinol 300mg daily was given for advanced hyperuricaemia with uncontrolled tophaceous growth, till the symptoms subsided and then maintenance dose of $100 \mathrm{mg}$ daily along with a regular scheduled Rheumatology follow up advised), a meticulous surgical excision was performed for bilateral elbow swellings by standard posterior approach (Figure 2). Careful dissection and meticulous separation of tophi from the underlying soft tissue external to the cubital tunnels. The tophi were external to the cubital tunnel but found compressing the nerve underneath and indirectly causing cubital tunnel compression neuropathy due to mass effect. Hence both the cubital tunnels were also decompressed to release the pressure off the compressed nerve. The nerves were not transposed and left in the anatomical location. The surgery was performed on bilateral elbows in the same sitting by two Orthopaedic surgeons and assistants. Histopathological examination was done, and diagnosed to be tophaceous gout. The tophi are displayed in Figure 3 below. Post-operatively patient had immediate reduction in symptoms and normal distal neurovascular status.

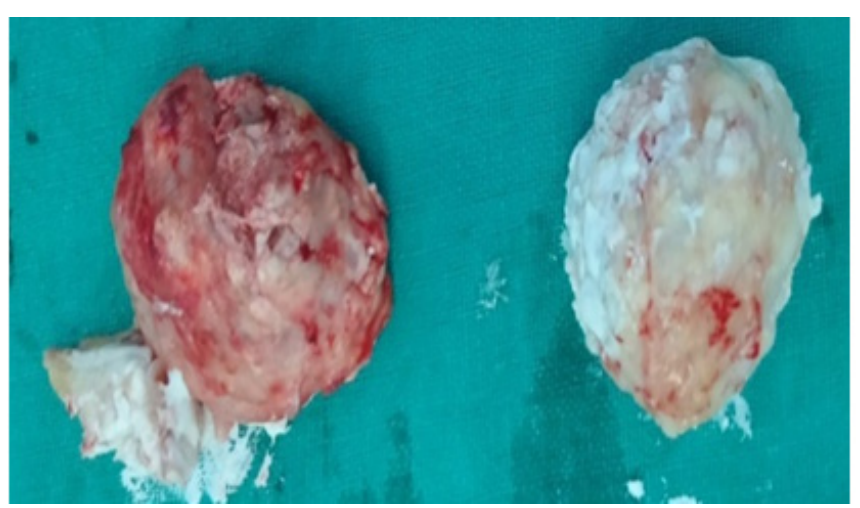

Figure 3 Tophi removed en-mass from both elbows and histopathological examination confirms gout deposits.

\section{Outcome and follow-up}

Paraesthesia and discomfort in elbows reduced substantially over six weeks and disappeared thereafter. Two year follow up revealed no recurrence. No joint stiffness was appreciated over this time. Occasional analgesia was required when the patient accidentally hit the tophaceous swellings on multiple small joints during manual labour. Over two years the swellings had reduced remarkably in all the affected joints (metatarsophalangeal joints of feet and bilateral elbows), and routine life activity was comfortably performed as per the patient. We do not have any further follow up.

\section{Discussion}

This case is a rare presentation with bilateral neural symptoms due to tophaceous gout around elbows. Tophi are often late presentations. Controlling serum uric acid levels below deposition threshold either by dietary or pharmaceutical drugs is the mainstay of management, resulting in dissolution of monosodium urate crystals. ${ }^{1,2} \mathrm{~A}$ recently published similar case report had also found gout as the cause of a large elbow soft tissue swelling that was initially approached as a pseudotumor or sarcomatous swelling. ${ }^{3}$ Extensor surfaces commonly develop tophi which is a pathognomonic feature of gout. ${ }^{4}$ There are no defined guidelines or rules of management when it comes to entrapment of neuro-vascular structures in chronic tophi. There are no prefixed guidelines pertaining to various tophi management especially in the scenario of compression signs. ${ }^{5}$ The best logical measure is to control further accumulation and careful surgical excision of the mass. There have been rare presentations of these tophi in the past as well like a 60 -year-old patient reported in 2001, with a tophi deposition in cervical spine leading to quadriparesis that later recovered after decompression; final result after more than one intervention being return to work. ${ }^{6}$ In an elderly male with carpal tunnel syndrome, on releasing the transverse carpal ligament surgically, gouty tophi were detected on the median nerve and another similar case with cubital tunnel signs published in 2017..$^{7,8}$

All these evidences indicate hyperuricemia is a not a definitive indicator of gout and the pathology may still exist in spite of normal serum urate levels. ${ }^{9}$ Gold standard is demonstration of differential crystals on polar microscopy. ${ }^{10,11}$ Other investigation modalities have also been proposed in literature as adjuncts as well as alternatives to conventional modalities. Few examples include Ultrasound, Computed tomography (CT), Dual energy CT (DECT), Magnetic resonance imaging, Doppler scan, PET (Positron Emission Tomography). Ultrasound is one of the most favoured modalities in view of many advantages like detection of active tophi with double contour sign in the articular cartilage ${ }^{12}$ and differentiation between hot and cold tophi. ${ }^{13}$ It can detect the crystal deposition on cartilage which may be missed by a DECT. Ultrasound depicts a snow storm appearance in synovial fluid upon pressurisation of overlying skin. ${ }^{14,15}$ It is an essential non-invasive modality of investigation that may be utilised for assessing disease progression, especially in recurrent scenarios and multiple loci where recognising an active tophi may be paramount. Controlling the level of serum uric acid is very essential in preventing recurrences and chronic tophi formation. It contributes and is directly related to disappearance of the pathognomonic features. ${ }^{16}$ Medical treatment in chronic gout is difficult to follow regularly in long term, ${ }^{1}$ but is very important in view of evidence relating duration of gout to the time needed for disappearance of synovial fluid crystals after medical treatment. ${ }^{17}$

Patient education and dietary changes are essential pillars of successful treatment and long-term control of serum uric acid levels. ${ }^{18-20}$

We acknowledge that this is a level 4 evidence and has the obvious associated risks of biases like reporting bias, publication bias and 
lack of generalisability. The readers should avoid overinterpretation of positive outcomes from a rare or atypical case scenario. The aim of such a case presentation, is to intrigue the readers into various complications possible with tophi around the neurovascular structures and sensitize the clinicians to detect and report similar cases.

\section{Learning points}

i. Although any soft tissue swelling around the joints could be gouty tophi, a thorough history and exclusion of differentials most likely in the pertinent clinical scenario must be done, before aggressive surgery.

ii. Untreated patients or failed medical treatment, may lead to persistent accumulation of the uric acid crystals in soft tissues leading to neurological symptoms from local mass compressing nerves in vicinity.

iii. Early intervention may prevent the long-term compression of nerves.

iv. Serum uric acid is not a reliable diagnostic measure for chronic tophi but controlled serum urate levels have a direct relation to reducing joint crystals in long term and hence the required regular medical treatment must never be ignored.

v. Follow-up is as important as the meticulous surgical excision of complete mass.

\section{Patient's perspective}

Patient education dis play a major role in controlling the symptoms in long run. Although the patient now regularly got his serum uric acid levels evaluated while on anti-hyperuricaemia drugs and was symptomless for 2 years after surgical excision, there was no further follow up or input available from him.

\section{Acknowledgments}

None.

\section{Conflicts of interest}

The authors declare no conflicts of interest.

\section{References}

1. Pascual E, Sivera F. Time required for disappearance of urate crystals from synovial fluid after successful hypouricaemic treatment relates to the duration of gout. Ann Rheum Dis. 2007;66(8):1056-1058.

2. Singh, Jasvinder, MBBS, MPH. Challenges Faced by Patients in Gout Treatment: A Qualitative Study. J clin rheumatol. 2014;20(3):172-174.

3. Jalan D, Maley D, Elhence A, et al. Massive Gouty Tophi Presenting as Pseudotumor of the Elbow: A Rare Presentation. Cureus. 2020;12(1):e6769.
4. Ragab G, Elshahaly M, Bardin T: Gout: an old disease in new perspective — a review. J Adv Res. 2017;8(5):495-511.

5. Peng-Bo Luo, Chang-Qing Zhang. Chronic carpal tunnel syndrome caused by covert tophaceous gout: A case report. World J Clin Cases. 2018;6(9):279-283.

6. E St George, C E Hillier, R Hatfield. Spinal cord compression: an unusual neurological complication of gout. Rheumatology. 2001;40(6):711-712.

7. Chuang Huo-Li, Wong Cheuk-Wah. Carpal Tunnel Syndrome Induced by Tophaceous Deposits on the Median Nerve: Case Report. Neurosurgery. 1994;34(5):919.

8. Skouteris D, Andritsos G, Tasios N, et al. Cubital Tunnel Syndrome Caused By Uric Acid Tophi: An Unusual Case. Ortho \& Rheum Open Access J. 2017;7(2):555707.

9. Badulescu M, Macovei L, Rezus E. Acute gout attack with normal serum uric acid levels. Rev Med Chir Soc Med Nat Iasi. 2014;118(4):942-945.

10. Underwood M. Diagnosis and management of gout. BMJ 2006;332(7553):1315-1319.

11. Chhana A, Dalbeth N. The gouty tophus: a review. Curr Rheumatol Rep. 2015;17(3):19

12. Gutierrez M, Smith W, Thiele R, et al. Defining elementary ultrasound lesions in gout. Preliminary results of Delphi consensus and web-exercise reliability. Ann Rheum Dis. 2014;73(Suppl 2):302.

13. Nestorova R, Fodor D. Crystal-induced arthritis. In: El Miedany Y, editor. Musculoskeletal ultrasonography in rheumatic diseases. Springer International Publishing; Cham. 2015. pp. 137-167.

14. Grassi W, Gutierrez M, Filippucci E. Chapter 16 - crystal-associated synovitis A2 - Wakefield, Richard J. In: D’Agostino MA, editor. Essential applications of musculoskeletal ultrasound in rheumatology. Content Repository Only; Philadelphia: 2010. pp. 187-197.

15. Filippucci E, Di Geso L, Grassi W. Tips and tricks to recognize microcrystalline arthritis. Rheumatol (Oxford, England). 2012.

16. Kiltz U, Smolen J, Bardin T, et al. Treat-to-target (T2T) recommendations for gout. Ann Rheum Dis. 2017;76(4):632-638.

17. Reach G. Treatment adherence in patients with gout. Joint Bone Spine. 2011;78(5):456-459.

18. Khanna D, Fitzgerald JD, Khanna PP, et al. 2012 American College of Rheumatology guidelines for management of gout. Part 1: systematic nonpharmacologic and pharmacologic therapeutic approaches to hyperuricemia. Arthritis Care Res (Hoboken) 2012;64(10):1431-1446.

19. Khanna D, Khanna PP, Fitzgerald JD, et al. 2012 American College of Rheumatology guidelines for management of gout. Part 2: therapy and anti inflammatory prophylaxis of acute gouty arthritis. Arthritis Care Res (Hoboken). 2012;64(10):1447-1461.

20. Richette P, Doherty M, Pascual E, et al. 2016 updated EULAR evidencebased recommendations for the management of gout. Ann Rheum Dis. 2017;76(1):29-42. 